\title{
KERNELS OF REPRESENTATIONS AND GROUP EXTENSIONS
}

\author{
I. SCHOCHETMAN
}

\begin{abstract}
We first determine the kernels of those unitary representations of a locally compact group which are obtained by integrating, inducing and tensoring. We then describe a broad class of group extensions for which the previous results can be used to find (1) the kernel of any representation and (2) necessary and sufficient conditions for these extensions to be maximally almost periodic.
\end{abstract}

Introduction. The primary purpose of this paper is to determine the kernel of any (unitary) representation of a reasonably general locally compact group extension. (See $\S 4$ for a specific description of the extension.) Roughly speaking, the representations of such an extension are obtained by successively tensoring, inducing and integrating (at least in the separable case) representations of certain subgroups. In $\S 1$ we determine the kernel of a direct integral. (This is the only place where we find it necessary to introduce separability assumptions.) This determination has the effect of reducing our problem to the consideration of irreducible representations only. In $\S 2$ we describe the kernel of an induced representation, thus extending Lemma 2.1 of [11] to the nonseparable case. Regarding the tensor product of representations, it is well known (see [9] and $[6, \S 17])$ that the most useful kernel to determine is that of the tensor product of projective representations or, more accurately, of representations of corresponding central group extensions of the circle. We do this in $\S 3$. Finally, in $\$ 4$ we describe the type of extension for which the preceding results solve the problem stated at the beginning. This section is essentially a combination and augmentation of R. J. Blattner's work in [3] and the relevant results of J. M. G. Fell in $\S 17$ of [6]. We conclude by indicating how the previous results can be used to find necessary and sufficient conditions for the extension to be maximally almost periodic.

Throughout this paper, $G$ will be a locally compact group and all representations will be unitary. If $h$ is a group homomorphism then

Received by the editors January 4, 1971.

AMS (MOS) subject classifications (1969). Primary 2265, 2260; Secondary 2220.

Key words and phrases. Locally compact group, unitary representation, kernel of a representation, direct integral representation, induced representation, tensor product representation, projective representation, group extension, central extension, maximally almost periodic.

c) American Mathematical Society 1973 
$\operatorname{ker}(h)$ will denote its kernel. In general we will not distinguish between an object (e.g. representation, $L^{2}$-function or group extension) and its equivalence class.

1. Direct integral representation. The first step in the solution of our problem is to determine the kernel of a direct integral. As general references for this section, we suggest [4, Chapter II, $\S \S 1-2]$ and $[\mathbf{5}, \S 8$ and Appendix A]. The following is easy to verify:

LEMMA 1. Let $\left\{T^{i}\right\}$ be an arbitrary family of representations of $G$. If $T$ is the direct sum $\oplus T^{i}$, then $\operatorname{ker}(T)=\bigcap \operatorname{ker}\left(T^{i}\right)$.

Now let $(Z, \mathscr{B}, \mu)$ be a $\sigma$-finite measure space and $\mathscr{N}$ the null sets in $\mathscr{B}$.

Lemma 2. Let $\mathscr{H}_{0}$ be a fixed separable Hilbert space. Suppose $G$ is second countable and $\left\{T^{z}: z \in Z\right\}$ is a measurable field of representations of $G$ in $\mathscr{H}_{0}$. Denote $L^{2}\left(Z, \mathscr{B}, \mu, \mathscr{H}_{0}\right)$ and $\int_{Z} T^{z} d \mu(z)$ by $\mathscr{H}$ and $T$ respectively. Then

$$
\operatorname{ker}(T)=\bigcup_{N \in \mathcal{A}^{-}} \bigcap_{z \in N^{\prime}} \operatorname{ker}\left(T^{z}\right),
$$

where $N^{\prime}$ is the complement of $N$ in $Z$.

Proof. Let $x \in \operatorname{ker}(T)$. Then for each $f$ in $\mathscr{H}$, we have $T_{x} f=f$ almost everywhere on $Z$. Since $\mu$ is equivalent to a finite measure, there is no loss in generality if we assume $0<\mu(Z)<\infty$. Suppose $\left\{v_{n}\right\}$ is a countable dense subset of $\mathscr{H}_{0}$. Then for each $n$, the function $f_{n}(z)=v_{n}, z \in Z$, belongs to $\mathscr{H}$ and $\left(T_{x} f_{n}\right)(z)=\left(T_{x}^{z}\right)\left(v_{n}\right)=v_{n}$ for $z$ outside some null set $N_{n}$. Let $N=\bigcup N_{n}$. Then $N$ belongs to $\mathscr{N}$ and $x$ belongs to

$$
\bigcap\left\{\operatorname{ker}\left(T^{z}\right): z \in N^{\prime}\right\} \text {. }
$$

This gives the forward inclusion. The reverse inclusion is straightforward.

THEOREM 1. Suppose $\left\{T^{z}: z \in Z\right\}$ is a measurable field of representations of $G$ in the corresponding measurable field of Hilbert spaces $\left\{\mathscr{H}^{z}: z \in Z\right\}$. Denote $\int_{Z} \mathscr{H}^{z} d \mu(z)$ and $\int_{Z} T^{z} d \mu(z)$ by $\mathscr{H}$ and $T$ respectively. Then once again $\operatorname{ker}(T)=\bigcup_{N \in \mathcal{M}} \bigcap_{z \in N^{\prime \prime}} \operatorname{ker}\left(T^{z}\right)$.

Proof. We first reduce the present problem to the one considered in Lemma 2. For each $p$ such that $1 \leqq p \leqq \aleph_{0}$, let $Z_{p}$ be the set of $z$ in $Z$ for which the dimension of $\mathscr{H} z$ is $p$. Note that $Z_{p}$ is in $\mathscr{B}$. Since the kernel of any representation depends only on its equivalence class, we may suppose $\mathscr{H}^{z}=\mathscr{H}_{p}$ for each $z$ in $Z_{p}$, where $\mathscr{H}_{p}$ is any $p$-dimensional Hilbert space. Then, letting $\mathscr{H}^{p}=L^{2}\left(Z_{p}, \mathscr{B}\left|Z_{p}, \mu\right| Z_{p}, \mathscr{H}_{p}\right)$, we see that

$$
\mathscr{H}^{p}=\int_{Z_{p}} \mathscr{H}^{z} d \mu(z) \text { and } \mathscr{H}=\oplus\left\{\mathscr{H}^{p}: 1 \leqq p \leqq \aleph_{c}\right\} .
$$


Similarly, if we let $T^{p}=\int_{Z_{p}} T^{z} d \mu(z)$, then

$$
T=\oplus\left\{T^{p}: 1 \leqq p \leqq \aleph_{0}, \mu\left(Z_{p}\right)>0\right\} .
$$

Therefore, by Lemma 1, we have

$$
\operatorname{ker}(T)=\bigcap\left\{\operatorname{ker}\left(T^{p}\right): 1 \leqq p \leqq \aleph_{0}, \mu\left(Z_{p}\right)>0\right\} .
$$

But by Lemma 2,

$$
\operatorname{ker}\left(T^{p}\right)=\bigcup_{N \in \hat{\mathcal{H}}_{p}} \bigcap_{z \in N^{\prime} \cap Z_{p}} \operatorname{ker}\left(T^{z}\right),
$$

where $\mathscr{N}_{p}=\mathscr{N} \mid Z_{p}$. Observe now that if $\mu\left(Z_{p}\right)=0$, then $Z_{p} \in \mathscr{N}_{p}$ and consequently $\operatorname{ker}\left(T^{p}\right)=G$ (the intersection is over an empty index set). Thus,

$$
\operatorname{ker}(T)=\bigcap\left\{\operatorname{ker}\left(T^{p}\right): 1 \leqq p \leqq \boldsymbol{\aleph}_{0}\right\} .
$$

Now we verify the set equality in the statement of the theorem.

Let $x \in \operatorname{ker}(T)$, i.e. $x \in \operatorname{ker}\left(T^{p}\right), 1 \leqq p \leqq \aleph_{0}$. Then for each $p$, there exists $N_{p}$ in $\mathscr{N}_{p}$ such that $x \in \bigcap\left\{\operatorname{ker}\left(T^{z}\right): z \in N_{p}^{\prime} \cap Z_{p}\right\}$. Hence, $N=\bigcup N_{p}$ belongs to $\mathscr{N}$ and $N^{\prime}=\bigcup\left(N_{p}^{\prime} \cap Z_{p}\right)$. Let $z \in N^{\prime}$, i.e. $z \in N_{p}^{\prime} \cap Z_{p}$, some $p$. Then necessarily, $x \in \operatorname{ker}\left(T^{z}\right)$, i.e. $x \in \bigcap\left\{\operatorname{ker}\left(T^{z}\right): z \in N^{\prime}\right\}$. Conversely, suppose $N \in \mathscr{N}$ and $x \in \bigcap\left\{\operatorname{ker}\left(T^{z}\right): z \in N^{\prime}\right\}$, i.e. $x \in \operatorname{ker}\left(T^{z}\right)$, for all $z \in N^{\prime}$. Then $N_{p}=N \cap Z_{p}$ is in $\mathscr{N}_{p}$ and $N=\bigcup N_{p}$ is a disjoint union. Once again $N^{\prime}=\bigcup\left(N_{p}^{\prime} \cap Z_{p}\right)$ so that

$$
\bigcap_{z \in N^{\prime}} \operatorname{ker}\left(T^{z}\right)=\bigcap_{1 \leqq p \leqq N_{0}} \bigcap_{z \in N_{p^{\prime}} \cap Z_{p}} \operatorname{ker}\left(T^{z}\right) .
$$

Hence, for each $p, x \in \bigcap\left\{\operatorname{ker}\left(T^{z}\right): z \in N_{n}^{\prime} \cap Z_{p}\right\}$, where $N_{p} \in \mathscr{N}_{p}$, i.e. $x \in \operatorname{ker}\left(T^{p}\right)$. This completes the proof.

Thus, in view of Théorème 8.5.2 of [5], the problem of determining the kernel of any representation of a locally compact group reduces (in the separable case) to the corresponding problem for any irreducible representation.

2. Induced representations. The next step is to determine the kernel of an induced representation. Let $H$ be a closed subgroup of $G$ and $T$ a representation of $H$. For the definition of $U^{T}$ we refer the reader to [2] or [7] (see [8] for the separable case). Let $\Delta_{G}$ (resp. $\Delta_{H}$ ) be the modular function for $G$ (resp. $H$ ) and $\delta=\Delta_{G} / \Delta_{I I}$.

THEOREM 2. The function $\delta$ is a continuous homomorphism of $H$ into the positive reals and

$$
\operatorname{ker}\left(U^{T}\right)=\left(\bigcap_{y \in G} y \operatorname{ker}(T) y^{-1}\right) \cap\left(\bigcap_{y \in G^{*}} y \operatorname{ker}(\delta) y^{-1}\right) .
$$


Proof. This is Lemma 2.1 of [11] in the nonseparable case. The proof of this lemma extends very naturally to the case at hand, so we leave the details to the reader.

3. Tensor product representation. The final consideration is the kernel of a certain type of tensor product which we now discuss. The general reference for this section is $[6, \S 17]$. The circle group will be denoted by $C$. Let $\xi: C \rightarrow_{i} H \rightarrow_{\sigma} G$ be a central $(C, G)$ group extension, $T$ a $\xi$-representation of $H$ in the Hilbert space $\mathscr{H}$. Let $U(\mathscr{H})$ be the topological group of unitaries on $\mathscr{H}, P U(\mathscr{H})$ the corresponding quotient of $U(\mathscr{H})$ modulo the circle group and $p: U(\mathscr{H}) \rightarrow P U(\mathscr{H})$ the canonical projection. Then $p T$ is a continuous homomorphism of $H$ into $P U(\mathscr{H})$ whose kernel contains $i(C)$, and $T$ gives rise to a continuous homomorphism $T^{0}$ of $G$ into $P U(\mathscr{H})\left(T^{0}\right.$ is a projective representation). We will denote $\operatorname{ker}\left(T^{0}\right)$ by $\operatorname{ker}_{\xi}(T)$. Thus, $\operatorname{ker}_{\xi}(T)$ is a closed normal subgroup of $G$ and is clearly equal to $\sigma(\operatorname{ker}(p T))$.

Now suppose $\xi_{k}: C \rightarrow_{i_{k}} H_{k} \rightarrow{ }_{\sigma_{k}} G$ is a central $(C, G)$ extension and $T^{k}$ is a $\xi_{k}$-representation of $H_{k}$ in the Hilbert space $\mathscr{H}_{k}$ for $k=1,2$. Then the outer tensor product $T^{1} \times T^{2}$ is a representation of $H_{1} \times H_{2}$ in $\mathscr{H}_{1} \times \mathscr{H}_{2}$. Furthermore, $H_{0}=\left\{\left(h_{1}, h_{2}\right) \in H_{1} \times H_{2}: \sigma_{1}\left(h_{1}\right)=\sigma_{2}\left(h_{2}\right)\right\}$ is a closed subgroup of $H_{1} \times H_{2}$ and $N_{0}=\left\{\left(i_{1}(c), i_{2}\left(c^{-1}\right)\right): c \in C\right\}$ is a closed normal subgroup of $H_{0}$. Let $\xi_{1} \xi_{2}: C \rightarrow_{i} H \rightarrow_{\sigma} G$ be the product central extension where $H=H_{0} / N_{0}$ (left cosets). Then it is easy to see that $N_{0} \subseteq \operatorname{ker}\left(T^{1} \times T^{2}\right)$ and consequently $\left(T^{1} \times T^{2}\right) \mid H_{0}$ passes to a representation of $H$ in $\mathscr{H}_{1} \otimes \mathscr{H}_{2}$ which we denote by $T^{1} \circledast T^{2}$; not surprisingly, this is a $\xi_{1} \xi_{2}$-representation of $G$. It is the " $\xi_{1} \xi_{2}$-kernel" of this kind of tensor product which turns out to be the most significant.

REMARK. If each $T^{k}$ is an ordinary representation of $G$, i.e. a representation of $C \times G$, then $T^{1} \ngtr T^{2}$ is the usual tensor product. If $G$ is second countable and $x_{k}$ is a multiplier corresponding to $\xi_{k}$ [1, pp. 24-25], then $T^{k}$ may be regarded as an $\alpha_{k}$-representation of $G$ and $T^{1} \otimes T^{2}$ is an $\alpha_{1} \alpha_{2}$-representation of $G$.

THEOREM 3. Let the notation be as above. Then

Proof. Let

$$
\operatorname{ker}_{\overline{5}_{1} \Sigma_{2}}\left(T^{1} \circledast T^{2}\right)=\operatorname{ker}_{\bar{\zeta}_{1}}\left(T^{1}\right) \cap \operatorname{ker}_{\bar{s}_{2}}\left(T^{2}\right) \text {. }
$$

$$
p: U\left(\mathscr{H}_{1} \otimes \mathscr{H}_{2}\right) \rightarrow P U\left(\mathscr{H}_{1} \underset{\otimes}{\otimes} \mathscr{H}_{2}\right) \text { and } p_{k}: U\left(\mathscr{H}_{k}\right) \rightarrow P U\left(\mathscr{H}_{k}\right) \text {, }
$$

$k=1,2$, be the canonical projections. Suppose $x \in \operatorname{ker}_{\bar{s}_{1} \dot{s}_{2}}\left(T^{1} \otimes T^{2}\right)$. Then there exists $h$ in $H$ such that $\sigma(h)=x$ and $h \in \operatorname{ker}\left(p\left(T^{1} \otimes T^{2}\right)\right)$. Consequently, there exists $\left(h_{1}, h_{2}\right)$ in $H_{0}$ for which $h=\left(h_{1}, h_{2}\right) N_{0}$ and

$$
\left(T^{1} \otimes T^{2}\right)_{h}=\left(T^{1} \times T^{2}\right)_{\left(h_{1}, h_{2}\right)}=T_{h_{1}}^{1} \otimes T_{h_{2}}^{2} .
$$


Since $p\left(\left(T^{1} \otimes T^{2}\right)_{h}\right)$ is trivial, it follows that $T_{h_{1}}^{1} \odot T_{h_{2}}^{2}$ must be a multiple of the identity $I_{1} \otimes I_{2}$ of $\mathscr{H}_{1} \otimes \mathscr{H}_{2}$. The only way this can happen is if $T_{h_{k}}^{k}$ is a multiple of $I_{k}$, i.e. $h_{k} \in \operatorname{ker}\left(p_{k} T_{k}\right), k=1,2$. But $x=\sigma(h)=$ $\sigma\left(\left(h_{1}, h_{2}\right) N_{0}\right)=\sigma_{1}\left(h_{1}\right)=\sigma_{2}\left(h_{2}\right)$, so that $x$ belongs to $\operatorname{ker}_{\xi_{1}}\left(T^{1}\right) \cap \operatorname{ker}_{\xi_{2}}\left(T^{2}\right)$. For the other inclusion, observe that each of the above steps is reversible.

4. The group extension. Let us now describe the kind of extension to which we referred in the introduction and see in particular how its irreducible representations are constructed. What follows is a summary of the main results of $[3]$ (see $[6, \S 16]$ also).

Suppose $H$ is a locally compact group, $N$ a closed normal subgroup, $N^{*}$ the primitive ideal space with the usual hull-kernel topology and $H \times N^{*} \rightarrow N^{*}$ the canonical transformation group. For each $z$ in $N^{*}$, let $H_{z}$ denote the corresponding (closed) stability subgroup and suppose $H / H_{z}$ (left cosets) is $\sigma$-compact. If $S$ is any representation of $N$, then $E_{S}$ will denote the Glimm projection-valued measure determined by $S$. Finally, if we assume that $N$ is regularly embedded in $H$, we have:

THEOREM 4. Let the notation be as above. Then there exists a mapping $\rho: \hat{H} \rightarrow N^{*} \mid H$ (orbit space) such that for $\theta \in N^{*} \mid H$, the mapping $T \rightarrow U^{T}$ is a bijection between $\left\{T \in \hat{H}_{z}:\{z\}\right.$ is $E_{T ! 1}$-thick $\}$ and $\rho^{-1}(\theta)$, where $z$ is any element of $\theta$.

It is not known if $\rho$ is onto in general. It will be if each $z$ in $N^{*}$ is semicompact, i.e. $N$ is type I (see [10] and [6, Proposition 17.3]). In any event, this theorem together with Theorem 2 reduces our problem to determining the kernel of a typical representation $T$ described in the last theorem. To assist in this task, we can refine the description of $T$ by making an additional assumption.

Lemma 3. Let $z$ in $N^{*}$ be semicompact and $S$ the unique element of $\hat{N}$ corresponding to $z$ [3, p. 1109]. If $T \in \hat{H}_{z}$, then $\{z\}$ is $E_{T \mid,-1}$ thick if and only if $T \mid N$ is a multiple of $S$.

Let us assume for the time being that $z$ and $S$ are fixed as in the lemma, $H_{z}=H, G=H \mid N$ and $\mathscr{H}$ is the Hilbert space of $S$. Then there exists $[6, \S 17]$ a central $(C, G)$ extension $\xi: C \rightarrow_{i} K \rightarrow_{\sigma} G$ constructed from $S$. Let us briefly review this construction in the group extension case. Define

$$
Q=\left\{(h, V) \in H \times U(\mathscr{H}): S_{l n n l-1}=V S_{n} V^{-1}, n \in N\right\} .
$$

Then $Q$ is a topological group, $M=\left\{\left(n, S_{n}\right): n \in N\right\}$ is a closed normal subgroup and $K=Q \mid M$. Let $\tau: Q \rightarrow K$ and $\pi: H \rightarrow G$ be the canonical projections and define $\beta: Q \rightarrow H$ by $\beta(h, V)=h$. Then $\sigma^{\prime}=\pi \beta$ is a continuous homomorphism and $\operatorname{ker}\left(\sigma^{\prime}\right) \supseteq M$. The resulting continuous homomorphism 
of $K$ into $G$ is $\sigma$ which is necessarily onto by our assumption. Define $j: C \rightarrow Q$ by $j(c)=(e, c I)$, where $e$ is the identity of $H$ and $I$ is the identity operator on $\mathscr{H}$. Then the mapping $i: C \rightarrow K$ is given by $i(c)=\tau(j(c))$. If $h$ is in $H$, the elements $V$ of $U(\mathscr{H})$ for which $(h, V) \in Q$ form a unique coset in $P U(\mathscr{H})$ which we will call $S_{h}^{\prime}$. Then $h \rightarrow S_{h}^{\prime}$ is a homomorphism of $H$ into $P U(\mathscr{H})$. Since $z$ is semicompact, $S^{\prime}$ is continuous and consequently, $\beta, \sigma^{\prime}$ and $\sigma$ are all open. It follows that $C \rightarrow_{j} Q \rightarrow{ }_{\beta} H$ is a central $(C, H)$ extension which we denote by $\pi \xi$.

Proposition 1. There is a one-to-one correspondence $W \leftrightarrow T$ between the $\xi^{-1}$-representations $W$ of $G$ and the representations $T$ of $H$ for which $T \mid N$ is a multiple of $S$.

Proof. This is Theorem 17.1 of [6] for group extensions. The correspondence $W \rightarrow T$ is given as follows. The Hilbert space of $T$ is the tensor product of the Hilbert spaces of $S$ and $W$, and for each $h$ in $H$ we have $T_{h}=V \otimes W_{\tau(h, V)}$, where $V$ is any element of $U(\mathscr{H})$ such that $(h, V)$ belongs to $Q$. The operator $T_{h}$ depends only on $h$. Notice that $W_{\tau}$ is a $(\pi \xi)^{-1}$ representation of $H$ and $S^{\prime}$ is a projective representation of $H$. For obvious reasons we will denote this hybrid representation by $S^{\prime} \times W_{\tau}$.

Proposition 2. Let the notation and assumptions be as above. Then

$$
\operatorname{ker}\left(S^{\prime} \otimes W \tau\right)=\operatorname{ker}\left(S^{\prime}\right) \cap \operatorname{ker}_{(\pi \xi-1}(W \tau) .
$$

Proof. The proof is similar to that of Theorem 3 above. We leave the details to the reader. Note that $\operatorname{ker}_{(\pi \xi)^{-1}}(W \tau)=\pi^{-1}\left(\operatorname{ker}_{\xi-1}(W)\right)$.

If it happens that there exists a $\pi \xi$-representation $S^{\prime \prime}$ of $H$ in $\mathscr{H}$ satisfying $p S^{\prime \prime}=S^{\prime} \beta$ (where $p: U(\mathscr{H}) \rightarrow P U(\mathscr{H})$ ), then $S^{\prime \prime} \otimes W_{\tau}$ is a legitimate representation of $H$ (as in $\S 3$ ), $S^{\prime \prime} \times W_{\tau}=S^{\prime} \times W \tau$, and by Theorem 3,

$$
\operatorname{ker}\left(S^{\prime} \times W \tau\right)=\operatorname{ker}_{\bar{n} \dot{\xi}}\left(S^{\prime \prime}\right) \cap \operatorname{ker}_{(\bar{\pi})^{-1}}(W \tau) .
$$

Observe that such $S^{\prime \prime}$ will exist if $H$ is second countable [1, Chapter 1, $\S 4]$.

As an application of the above consider the following. In Theorem 2.6 of [11] we gave necessary and sufficient conditions for the extension $H$ (as in Theorem 4) to be maximally almost periodic provided it was second countable. The discussion there can be naturally enlarged so as to do the same here. Specifically, if $H$ is as above, then condition (ii) of Theorem 2.6 (interpreted in the more or less obvious way) is equivalent to the maximal almost periodicity of $H$. If we also assume $N$ is type $\mathrm{I}$, then condition (iii) of Theorem 2.6 is also equivalent. Finally, the two corollaries following this theorem are also valid. We leave the verifications of these claims to the interested reader. 


\section{REFERENCES}

1. L. Auslander and C. C. Moore, Unitary representations of solvable Lie groups, Mem. Amer. Math. Soc. No. 62 (1966). MR 34 \#7723.

2. R. J. Blattner, On induced representations, Amer. J. Math. 83 (1961), 79-98. MR 23 \#A2757.

3. - Group extension representations and the structure space, Pacific J. Math. 15 (1965), 1101-1113. MR 32 \#5785.

4. J. Dixmier, Les algèbres d'opérateurs dans l'espace hilbertien (Algèbres de von Neumann), Cahiers scientifiques, fasc. 25, Gauthier-Villars, Paris, 1957. MR 20 \#1234.

5. - Les $C^{*}$-algèbres et leurs représentations, Cahiers scientifiques, fasc. 19, Gauthier-Villars, Paris, 1964. MR 30 \#1404.

6. J. M. G. Fell, An extension of Mackey's method to Banach *-algebraic bundles, Mem. Amer. Math. Soc. No. 90 (1969). MR 41 \#4255.

7. F. P. Greenleaf, Amenable actions of locally compact groups, J. Functional Analysis 4 (1969), 295-315. MR 40 \#268.

8. G. W. Mackey, Induced representations of locally compact groups. I, Ann. of Math. (2) 55 (1952), 101-139. MR 13, 434.

9. - Unitary representations of group extensions. I, Acta Math. 99 (1958), 265-311. MR 20 \#4789.

10. S. Sakai, On type I $C^{*}$-algebras, Proc. Amer. Math. Soc. 18 (1967), 861-863. MR 35 \#4741.

11. I. Schochetman, Dimensionality and the duals of certain locally compact groups, Proc. Amer. Math. Soc. 26 (1970), 514-520. MR 42 \#418.

Department of Mathematics, Oakland University, Rochester, Michigan 48063 